\title{
Magnetic Circular Dichroism in Electron Microscopy
}

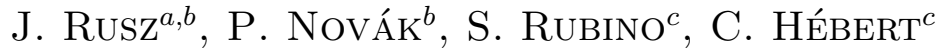 \\ AND P. SCHATtSCHNEIDER ${ }^{c}$ \\ ${ }^{a}$ Department of Physics, Uppsala University \\ P.O. Box 530, 75121 Uppsala, Sweden \\ ${ }^{b}$ Institute of Physics, Academy of Sciences of the Czech Republic \\ Na Slovance 2, 18221 Prague, Czech Republic \\ ${ }^{c}$ Institute for Solid State Physics, Vienna University of Technology \\ Wiedner Hauptstr. 8-10/138, 1040 Vienna, Austria

\begin{abstract}
Although the analogies between the electron energy loss spectra and X-ray absorption spectra were recognized long ago, the possibility to observe the magnetic circular dichroism in the electron microscope was demonstrated only recently. This technique combines the atom specificity with the high spatial resolution of the transmission electron microscope. Being a very young technique, there are still many open questions concerning the optimization of the signal to noise ratio. In this paper we study the dichroic signal dependence on the sample thickness, acceleration voltage and incoming beam tilt angle of bcc-Fe and fcc-Ni.
\end{abstract}

PACS numbers: 75.40.Mg, 78.20.Bh, 79.20.Uv

\section{Introduction}

The analogy between X-ray absorption spectroscopy (XAS) and electron energy loss spectroscopy (EELS) $[1,2]$ is based on the similar role of the polarization vector $\boldsymbol{\epsilon}$ in XAS and momentum transfer vector $\hbar \boldsymbol{q}$ in EELS. This has facilitated the detection of magnetic linear dichroism in the transmission electron microscopy (TEM) [3]. However the counterpart of X-ray magnetic circular dichroism (XMCD) [4-6] experiments with electron probes was thought to be technically impossible due to the low intensity of existing spin polarized electron sources. XMCD is an important technique providing atom-specific information about the magnetic properties of materials. Particularly the near edge spectra, where a well localized strongly bound electron with nonzero $l$ is excited to an unoccupied band state, allow to measure spin and orbital moments. Soon after 
the proposal of an experimental setup for detection of circular dichroism using a standard non-polarized electron beam in the TEM [7] we have demonstrated that such experiments (called energy-loss magnetic chiral dichroism, EMCD) are indeed possible [8]. This novel technique is of considerable interest for nanomagnetism and spintronics thanks to the high spatial resolution of the TEM. However, its optimization involves many open questions. Newly proposed experimental geometries lead to significantly improved signal to noise ratios and at the same time reduce the lateral resolution down to $10 \mathrm{~nm}$ or below.

\section{The principle of EMCD}

In both XMCD and EMCD experiments, the underlying process is an inelastic collision between the incident particle and an electron in the sample. In the near-edge spectra we study collisions with core electrons, i.e. low-lying well localized electrons from fully occupied shells (e.g. $2 p$-electrons in transition elements $\mathrm{Fe}, \mathrm{Co}$, or $\mathrm{Ni}$ ). These are excited into some unoccupied valence states above the Fermi level. Therefore both energy-loss near edge spectra (ELNES) and X-ray absorption near edge spectra (XANES) are probing the unoccupied density of states. The dominant transitions are the dipole allowed ones, where the orbital quantum number of the excited electron changes by 1 (e.g. $2 p \rightarrow 3 d$ in $\mathrm{Fe}, \mathrm{Co}$, and $\mathrm{Ni}$ ).

The EMCD experiment is essentially a standard ELNES experiment performed at particular experimental conditions. In XMCD, the circularly polarized incoming X-ray beam is a coherent superposition of two perpendicular and phase shifted linear polarizations $\left(\epsilon_{1} \pm \mathrm{i} \epsilon_{2}\right.$ with $\epsilon_{1} \perp \epsilon_{2}$ and the imaginary unit i signifying a phase shift of 90 degrees). The XMCD signal is a difference between the two XANES spectra measured for right-handed ("+" sign) and left-handed ("-" sign) circular polarizations. In EMCD we set the relative orientation of the sample and incoming beam in such a way, that the fast electron inside the crystal can be described as a coherent superposition of two Bloch waves $\boldsymbol{k}_{\mathrm{i}}$ and $\boldsymbol{k}_{\mathrm{i}}+\boldsymbol{G}$. This is called a two-beam case. The effective phase shift of these two Bloch wave components is determined by the experimental geometry and the dynamical diffraction effects. The detector is placed in the direction $\boldsymbol{k}_{\mathrm{f}}$, so that the wave transfer vectors $\boldsymbol{q}=\boldsymbol{k}_{\mathrm{f}}-\boldsymbol{k}_{\mathrm{i}}$ and $\boldsymbol{q}^{\prime}=\boldsymbol{k}_{\mathrm{f}}-\boldsymbol{k}_{\mathrm{i}}-\boldsymbol{G}$ are perpendicular $\left(\boldsymbol{q} \perp \boldsymbol{q}^{\prime}\right)$. There are two such detector positions and the EMCD signal is a difference of these two ELNES spectra $[7,8]$, as was the case for the XMCD experiment. The experimental setup of the EMCD is sketched in Fig. 1, where the two detector positions are denoted as $(+)$ and $(-)$ in the diffraction plane; they correspond to excitations with opposite chirality.

The theoretical description is thus based on (i) dynamical theory of electron diffraction and (ii) ab initio calculations of the electronic structure. The dynamical diffraction theory describes the movement of fast electrons through the sample, while the electronic structure calculations are necessary to calculate the matrix elements of the inelastic transitions. A detailed description of our theoretical approach was recently published in Ref. [9]. 


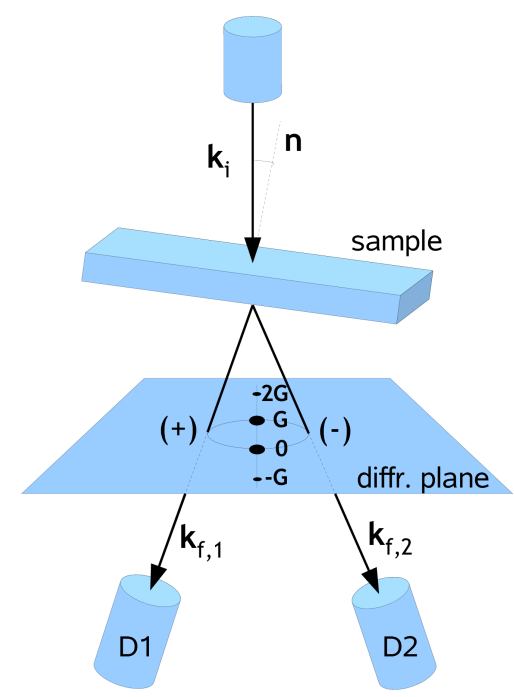

Fig. 1. Experimental geometry. The bar shaped sample is tilted so that its surface normal $\boldsymbol{n}$ (corresponding to a zone axis) forms an angle of approximately 10 degrees with respect to the incoming beam direction $\boldsymbol{k}_{\mathrm{i}}$. This excites a systematic row of Bragg reflections $-\boldsymbol{G}, \mathbf{0}, \boldsymbol{G}, 2 \boldsymbol{G}, \ldots$ Two spectra are measured by the detectors D1 and D2 placed in the $\boldsymbol{k}_{\mathrm{f}, 1}$ and $\boldsymbol{k}_{\mathrm{f}, 2}$ directions on the Thales circle above the strongest ( 0 and $\boldsymbol{G}$ ) reflections in the diffraction plane. The two-beam case is obtained by setting the Laue circle center to $\boldsymbol{G} / 2$.

In all calculations presented in this work we used the following treatment: (i) for the dynamical diffraction theory part of the problem, we worked within the systematic row approximation with up to 10 diffracted beams for both incoming and outgoing beam. The detector position, orientation of the sample with respect to the incoming beam and the thickness of the sample were taken into account in our simulations. (ii) For the electronic structure calculations we have applied the density functional theory [10] within the local density approximation [11]. We used experimental values of the lattice parameters of all studied systems. The state-of-the-art full-potential linearized augmented plane waves code WIEN2k [12] was used with 40,000 $\boldsymbol{k}$-points in the first Brillouin zone and more than 100 basis functions per atom.

\section{Results}

To produce the two-beam case, the incoming beam is tilted from some highsymmetry direction by a few degrees. The direction of this tilt determines which excitations ( $\boldsymbol{G}$ vectors) will lie close to the Ewald sphere. Setting the Laue circle center (LCC) to $\boldsymbol{G} / 2$ leads to the two-beam case, in which the direct beam $\boldsymbol{k}_{\mathrm{i}}$ and the diffracted beam $\boldsymbol{k}_{\mathrm{i}}+\boldsymbol{G}$ have the same Bloch wave coefficients. Our simulations 
have shown that the dichroic signal is very sensitive to the selection of a particular systematic row. In Fig. 2 we show calculations of the thickness profiles (dependence of the signal on the sample thickness) of bcc-Fe and fcc-Ni for two $\boldsymbol{G}$ vectors. While $\boldsymbol{G}=(200)$ for iron and (220) for nickel display a broad peak around 15$20 \mathrm{~nm}$, setting $\boldsymbol{G}$ to (110) for iron and (200) for nickel leads to high-frequency oscillations with respect to thickness. This imposes more stringent requirements on specimen drift, uniformity of thickness in the sampled area, and precision of the determination of the thickness.
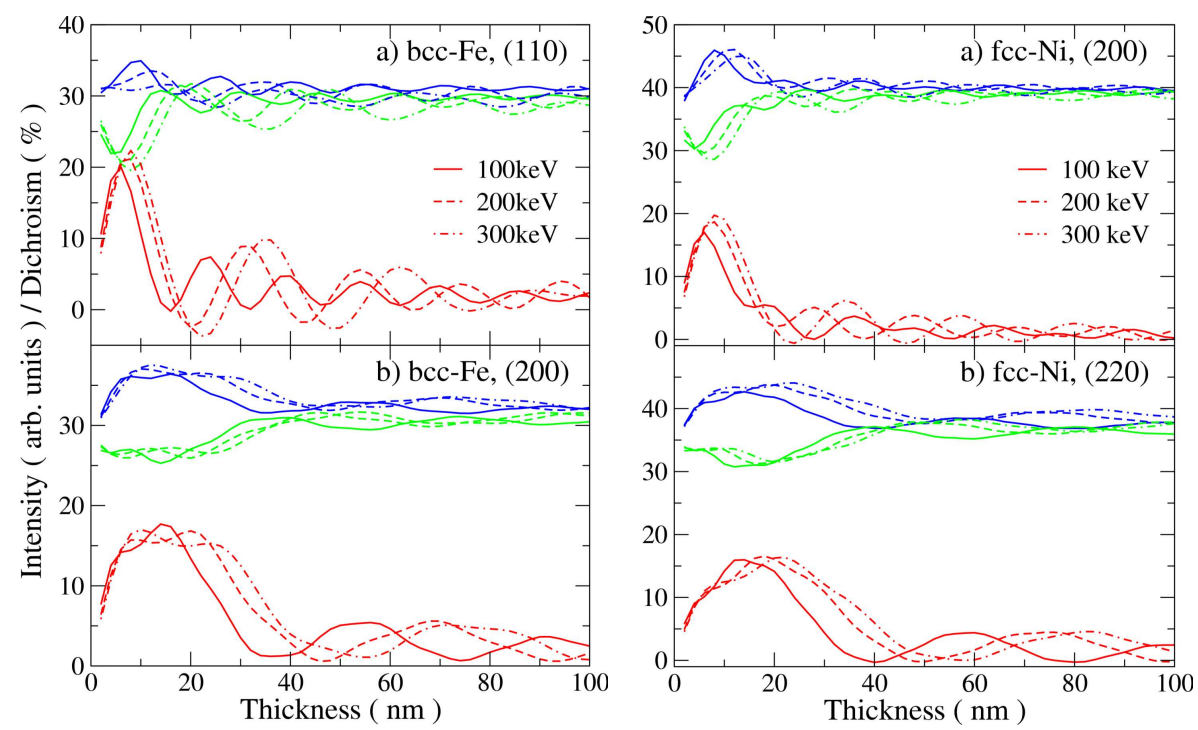

Fig. 2. Thickness profiles of bcc-Fe (left) and fcc-Ni (right) as a function of the acceleration voltage. Within each part, the top sets of curves (black and gray) correspond to the ELNES signal at the $L_{3}$ edge for the two detector positions (top and bottom of the Thales circle). These are given in arbitrary units. The bottom curves are the relative dichroic signal $\left(\sigma_{+}-\sigma_{-}\right) /\left(\sigma_{+}+\sigma_{-}\right)$in percent. Systematic row indices are (a) $\boldsymbol{G}=(110)$ and (b) $\boldsymbol{G}=$ (200) for iron and (a) $\boldsymbol{G}=$ (200) and (b) $\boldsymbol{G}=(220)$ for nickel, respectively.

Figure 2 simultaneously shows the dependence of the signal on the acceleration voltage of the fast electron. The general shape of the profiles is preserved, but non-negligible differences can be evidenced, especially with respect to the periodicity. For higher acceleration voltage the frequency of the oscillations in thickness decreases. The overall strength of the dichroic effect is not changed. This behavior can be qualitatively understood by observing that the fast electron mean free path increases when the acceleration voltage is increased and so does the extinction distance for each $\boldsymbol{G}$-reflection.

The next subject of our studies is the sensitivity of the signal on the beam tilt. We performed calculations of the thickness profiles of bcc-Fe for $\boldsymbol{G}=(110)$ 

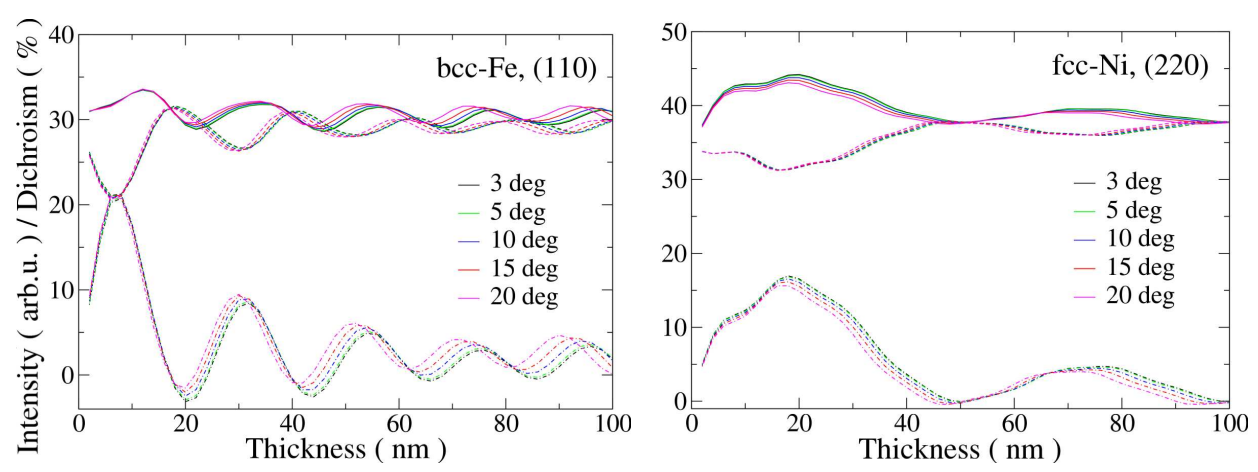

Fig. 3. Thickness profiles of bcc-Fe [left, $\boldsymbol{G}=(110)]$ and fcc-Ni [right, $\boldsymbol{G}=(220)]$ as a function of the incoming beam tilt angle at $200 \mathrm{keV}$ acceleration voltage. The full and dashed lines correspond to the ELNES signal at the $L_{3}$ edge for the two detector positions (top and bottom of the Thales circle). The dash-dotted line is the relative dichroic signal in percent.

and fcc-Ni for $\boldsymbol{G}=(220)$, respectively, at $200 \mathrm{keV}$ acceleration voltage as a function of the beam tilt from the [001] high-symmetry direction, see Fig. 3. The tilt angle in our calculations ranged from 3 to 20 degrees. It should be noted that for very low angles the systematic row approximation becomes questionable, because other reflections from the (001) plane outside the systematic row are also very close to the Ewald sphere and should no longer be neglected. However, this goes beyond the scope of this paper. For higher beam tilts the approximation is very good and, as we see from the figure, the influence of the beam tilt angle on the signal is very weak. Essentially, a larger tilt angle makes the oscillation period slightly smaller (this is connected with a smaller projection of the incoming beam wave vector to the (001) surface normal).

\section{Conclusions}

We have calculated the thickness profiles of bcc-Fe and fcc-Ni as a function of the acceleration voltage and the beam tilt. Higher acceleration voltages increase the period of so-called pendellösung oscillations - i.e. the variations of the signal intensity with thickness. Thus measurements at higher acceleration voltages are less sensitive to thickness variations in the sample, whereas the general shape of the thickness profiles remains the same. On the other hand, the sensitivity of the signal to the beam tilt is very weak. Larger tilt angles slightly reduce the pendellösung oscillation period.

\section{References}

[1] A.P. Hitchcock, Jpn. J. Appl. Phys. 32, 176 (1993).

[2] J. Yuan, N.K. Menon, J. Appl. Phys. 81, 5087 (1997). 
[3] P.A. van Aken, S. Lauterbach, Phys. Chem. Minerals 30, 469 (2003).

[4] B.T. Thole, P. Carra, F. Sette, G. van der Laan, Phys. Rev. Lett. 68, 1943 (1992).

[5] S.W. Lovesey, S.P. Collins, X-Ray Scattering and Absorption by Magnetic Materials, Clarendon Press, Oxford, UK 1996.

[6] J. Stöhr, J. Electron Spectrosc. Relat. Phenom. 75, 253 (1995).

[7] C. Hébert, P. Schattschneider, Ultramicroscopy 96, 463 (2003).

[8] P. Schattschneider, S. Rubino, C. Hébert, J. Rusz, J. Kuneš, P. Novák, E. Carlino, M. Fabrizioli, G. Panaccione, G. Rossi, Nature 441, 486 (2006).

[9] J. Rusz, S. Rubino, P. Schattschneider, Phys. Rev. B 76, 060408(R) (2007).

[10] P. Hohenberg, W. Kohn, Phys. Rev. 136, B864 (1964); W. Kohn, L.J. Sham, Phys. Rev. 140, A1133 (1965).

[11] J.P. Perdew, Y. Wang, Phys. Rev. B 45, 13244 (1992).

[12] P. Blaha, K. Schwarz, G.K.H. Madsen, D. Kvasnicka, J. Luitz, WIEN2k, Vienna University of Technology, Wien 2001. 